\title{
Post MI pericarditis or recurrent infarction-Diagnostic dilemma
}

\author{
Sachin Sondhi*, Kunal Mahajan, Ayushi Mehta and Munish dev \\ Department of Cardiology, Indira Gandhi Medical College, Shimla, Himachal Pradesh, India
}

\begin{abstract}
There are many causes of ST segment elevation in multiple leads in electrocardiogram. Differentiation is based on history, clinical examination and most importantly on electrocardiogram features like nature of ST segment elevation, leads specific ST segment elevation, reciprocal changes, presence or absence of Q wave, PR segment depression and ST/T wave ratio in V6. We report a case of 57 year male, presented with acute coronary syndrome in form of ST elevation inferolateral myocardial infarction and subsequently on day 3rd he developed chest pain, the diagnosis of post myocardial infarction pericarditis was made based on specific electrocardiogram findings.
\end{abstract}

\section{Introduction}

Pericardial inflammation after myocardial infarction can be either acute seen after 3 to 10 days after large transmural myocardial infarction, termed as peri-infarction pericarditis or immune mediated inflammation after 1 to 8 weeks termed as post myocardial infarction syndrome (Dressler Syndrome). The pain of pericarditis may be confused that resulting from post infarction angina or recurrent infarction. Characteristic nature of pain, presence of pericardial rub helps to differentiate these conditions but sensitivity is low. Electrocardiogram is a valuable tool for differentiation.

\section{Case summary}

57 year old male with no previous co morbidity presented to our department with history of acute onset crescendo angina of duration 8 hours. At time of presentation his blood pressure was $104 / 70 \mathrm{mmHg}$, pulse rate $104 / \mathrm{min}$. JVP was not raised and in chest there were no crepts. His ECG (Figure 1) showed $3 \mathrm{~mm}$ ST segment elevation in lead I, II, III, $\mathrm{aVF}$ and in lead V4,V5 and V6. There is reciprocal depression in lead $\mathrm{V} 1, \mathrm{~V} 2$ and V3.His cardiac troponin $\mathrm{T}$ was positive. The diagnosis of ST elevated inferolateral myocardial infarction was made and he was thrombolysed with streptokinase and then started on dual antiplatlets, beta blockers, statins and anticoagulation. His repeat ECG (Figure 2) after thrombolysis revealed ST segment elevation of only $1 \mathrm{~mm}$ and $\mathrm{T}$ wave inversion in inferior leads and in lead V6. 2D echocardiography revealed hypokinesia of inferolateral wall with ejection fraction of $42 \%$ and there was no evidence of mechanical complications. On day $3^{\text {rd }}$, he was again complaining of chest pain, which was increasing on coughing, on deep inspiration and on lying down. His blood investigations including complete blood counts, liver function tests, renal function tests and Chest $\mathrm{X}$ ray were normal. There was no pericardial rub and repeat ECG (Figure 3) revealed diffuse concave ST segment elevation in all precordial and limb leads except avR which showed ST depression. Initially possibility of reinfarction was kept in new territory. Pain was not relieved with nitrates. On close inspection of ECG there was diffuse concave ST segment elevation except avR which showed ST depression, PR segment depression seen in precordial leads along with PR segment elevation in lead avR and ST/T ratio in V6 was greater than 0.25 . All these features are suggestive of pericarditis. His echo done again which revealed mild circumferential pericardial effusion of thickness $3 \mathrm{~mm}$. Anticoagulation was stopped and he was started on aspirin $600 \mathrm{mg}$ 6 hourly. He responded to treatment and discharged on $6^{\text {th }}$ day. At the two-week follow-up visit, the patient was doing well.

\section{Discussion}

Post-MI pericarditis also termed as peri-infarction pericarditis is a common complication (25-40\% of patients with MI) and occurs early, within 3 to 10 days after the MI. Pain of pericarditis is central, increased on deep inspiration and on lying down, relieved on sitting and leaning forward, characteristically radiates to trapezius ridge and not relieved by nitrates. Physical examination may reveal the pathognomonic finding for pericarditis: the pericardial friction rub but at times, the rub may be evanescent [1]. It is important to distinguish pericarditis pain from pain of recurrent infarction as management differs in both.

ECG is valuable tool to differentiate between these two conditions. Changes on ECG classically occur in four stages. Not all cases of pericarditis include each of these four stages [2,3].

Stage I- typically occurs during the first few days of pericardial inflammation and is mainly characterized by diffuse ST-segment elevation. This stage may last up to two weeks.

Stage II- is characterized by return of the ST segments to baseline and flattening of the $\mathrm{T}$ wave and may last from days to several weeks.

Stage III- usually begins at the end of the second or third week and is characterized by inversion of the $\mathrm{T}$ waves in the opposite direction of the ST segment; this stage may last several weeks.

Correspondence to: Sachin Sondhi, Department of Cardiology, Indira Gandhi Medical College, Shimla, Himachal Pradesh, India, Tel: +91-8219508161; E-mail: ssachin119@gmail.com

Key words: Post MI pericarditis, post infarction angina, Electrocardiogram

Received: August 04, 2017; Accepted: August 23, 2017; Published: August 26, 2017 
Table 1. Shows common differentials of diffuse ST segment elevation in ECG and there differentiating features. "This is calculated by dividing the millimeters of ST-segment elevation by

\begin{tabular}{|c|c|c|c|}
\hline ECG findings & Acute pericarditis & Myocardial infarction \\
\hline ST segment shape & concave & convex \\
\hline Q waves & absent & present & present \\
\hline Reciprocal ST-segment changes & absent & Absent & Absent \\
\hline Location of ST-segment elevation & Limb and precordial leads & N/A involved artery \\
\hline ST/T ratio in lead V6 & $>0.25$ & absent & Precordial leads \\
\hline PR elevation and ST depression in avR & present & absent \\
\hline
\end{tabular}

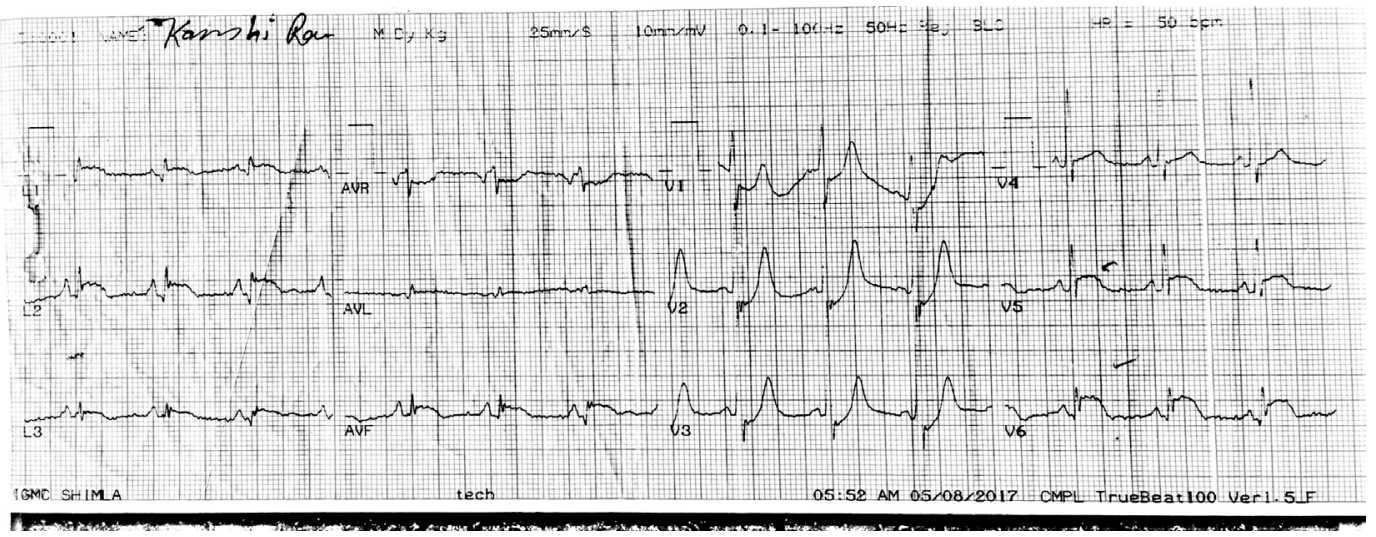

Figure 1. ECG on day $1^{\text {st }}$, showing 3mm STsegment elevation I, II, III, aVF and in lead V4,V5 and V6 along with reciprocal ST depression in lead V1, V2 and V3.

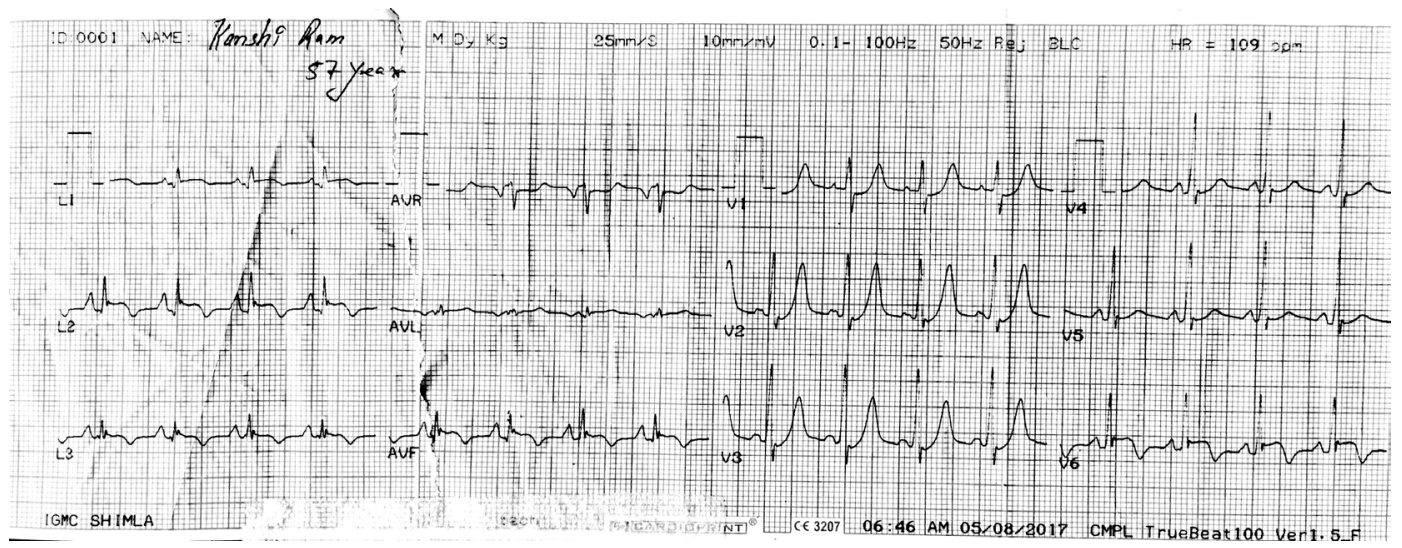

Figure 2. ECG after thrombolysis, showing $1 \mathrm{~mm}$ ST segment elevation with T wave inversion in lead I, II, III, aVF and in lead V6.

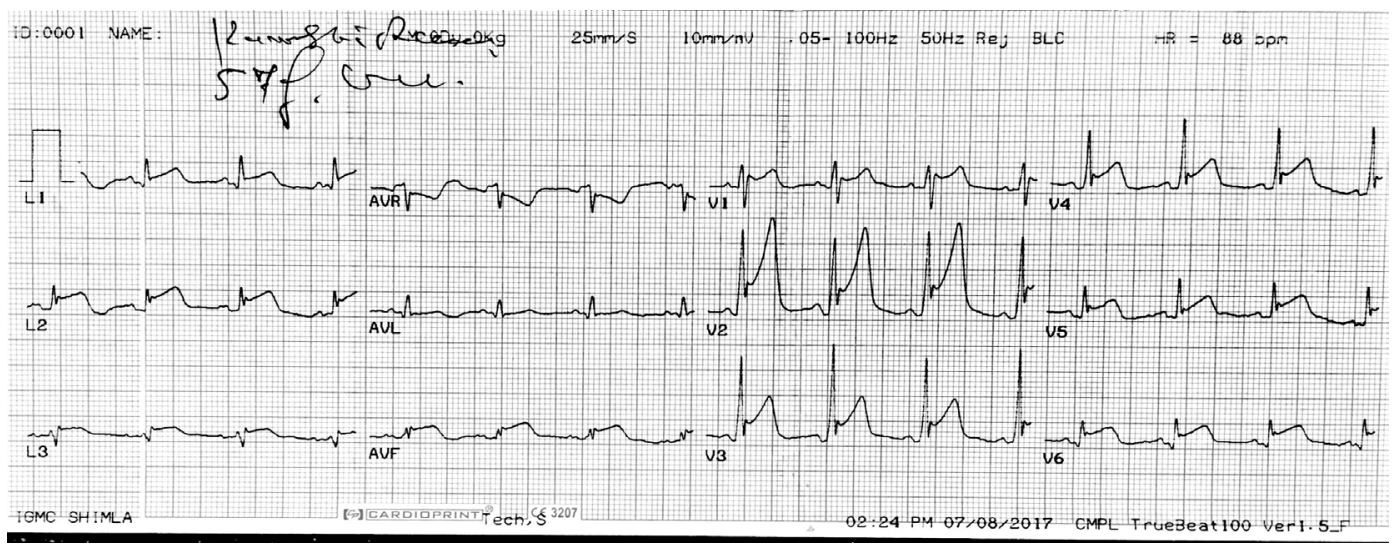

Figure 3. ECG showing diffuse concave ST segment elevation in all precordial leads and all limb leads except avR, which showed ST depression. Note PR segment depression in all precordial leads (best seen V2,V3 and V4) and PR elevation in Lead avR. ST/T ration in V6 is more than 0.25. All these features are characteristic of acute Pericarditis. 
Stage IV-represents the gradual resolution of the T-wave changes and may last up to three months

The most sensitive ECG change characteristic of acute pericarditis is ST-segment elevation, which reflects the abnormal repolarization that develops secondary to pericardial inflammation. There may also be ST-segment depression in leads aVR and V1. Typically, there are no changes during depolarization, so in the absence of underlying cardiac disease, the P wave and QRS complexes are normal. Depression of the $\mathrm{PR}$ segment is very specific of acute pericarditis and is attributed to subepicardial atrial injury and occurs in all leads except aVR and V1. These leads may exhibit PR-segment elevation $[1,4]$.

The pattern of ST-segment elevation is important in the diagnosis of acute pericarditis. The ST-segment elevation that occurs during acute pericarditis is usually "concave," compared with the "convex" appearance of the ST segment that occurs during the acute injury stage of a myocardial infarction. Another important feature of acute pericarditis is the widespread ST-segment elevation not corresponding with any specific arterial territory, which usually occurs in association with acute myocardial infarction [1]. Also, reciprocal changes are absent in acute pericarditis, although they are frequently found with acute myocardial infarction. The ST segments in acute pericarditis return to baseline in a few days and are followed by diffuse T-wave inversion, in conjunction with the ST segment at baseline [3].

Another feature that may aid in differentiating acute pericarditis from acute myocardial infarction is the absence of $\mathrm{Q}$ waves and the absence of T-wave inversion at the time of ST-segment elevation, both of which classically occur with acute myocardial infarction. Loss of $\mathrm{R}$-wave progression may occur with acute myocardial infarction, but this feature is not present with acute pericarditis [1].

Early repolarization is another benign variant associated with diffuse and concave ST segment elevation that occurs commonly in young males, especially blacks, and does not evolve with the stages of acute pericarditis [1]. Early repolarization is distinguished by STsegment elevation limited to the precordial leads, elevation of the ST segment in V1, an isoelectric ST segment in lead V6, notching of the terminal aspect of the QRS complex, and a shift to baseline of the ST segments with exercise [3]. Another useful clue in differentiating acute pericarditis from early repolarization is the ST/T ratio in lead V6. This is calculated by dividing the millimetres of ST-segment elevation by the millimetres to the tallest point of the T wave. Each value is measured from the isoelectric point. An ST/T ratio of greater than 0.25 in lead V6 suggests acute pericarditis [5]. Table 1 illustrates major differences based on ECG in between acute pericarditis, recurrent infarction and early repolarization variant.

Treatment involves discontinuation of anticoagulation only if there was presence of pericardial effusion, asprin $650 \mathrm{mg}$ every 4-6 hourly. Steroids and NSAIDS should be avoided because they interfere with myocardial scar formation [6].

\section{Learning points}

Diffuse concave ST segment elevation in all leads except avR, absent reciprocal changes, absent $\mathrm{Q}$ wave, PR segment depression in precordial leads and ST segment depression along with PR segment elevation in avR are important diagnostic clues for acute pericarditis.

\section{References}

1. Shabetai R (1990) Acute pericarditis. Cardiol Clin 8: 639-44. [Crossref]

2. Chou TC (1991) Electrocardiography in clinical practice. ( $3^{\text {rd }}$ edn). Philadelphia: Saunders, pp: 219-234.

3. Friedman HH (1985) Diagnostic electrocardiography and vectorcardiography. (3 $3^{\text {rd }}$ edn). New York: McGraw-Hill, pp: 320-327.

4. Spodick DH (1973) Diagnostic electrocardiographic sequences in acute pericarditis Significance of PR segment and PR vector changes. Circulation 48: 575-580. [Crossref]

5. Ginzton LE, Laks MM (1982) The differential diagnosis of acute pericarditis from the normal variant: new electrocardiographic criteria. Circulation 65: 1004-1009. [Crossref]

6. Imazio M, Spodick DH, Brucato A, Trinchero R, Adler Y (2010) Controversial issues in management of pericardial diseases. Circulation 121: 916-928. [Crossref]

Copyright: (C2017 Sondhi S. This is an open-access article distributed under the terms of the Creative Commons Attribution License, which permits unrestricted use, distribution, and reproduction in any medium, provided the original author and source are credited. 